DOI $10.15421 / 4221002$

УДК 624.13

\author{
С. В. Босаков ${ }^{1}$, д-р техн. наук, О. В. Козунова ${ }^{2}$ канд. техн. наук
}

\title{
СТАТИЧЕСКИЙ РАСЧЕТ ШАРНИРНО-СОЕДИНЕННЫХ БАЛОК НА УПРУГОМ СЛОЕ
}

Статический расчет шарнирно-соединенных балок на упругом однородном изотропном слое, как и на других моделях упругого основания, выполнен смешанным методом строительной механики и способом Жемочкина в случаях сосредоточенной и равномерно-распределенной нагрузок, действующих на балку с учетом ее собственного веса. Предложен универсальный подход для расчета шарнирно-соединенных балок на упругом слое, шарнирно соединенном с недеформируемым основанием, который позволяет с единых позиций рассчитывать шарнирно-соединенные балки любой жесткости при произвольной вертикальной нагрузке. Численная реализация предлагаемого подхода выполнена при помощи компьютерной программы В пакете Mathematica.

Ключевые слова: статический расчет; упругий слой; способ Жемочкина; смешанный метод строительной механики.

Актуальность проблемы. Обзор литературы. Теория расчета шарнирно-соединенных конструкций на упругом основании до настоящего времени не создана в полной мере. Исследованиями по расчету шарнирно-соединенных балок и плит на упругом основании ранее занимались следующие ученые: Б.Г. Коренев [11], Г.Я. Попов [12], И.А. Симвулиди [15], Р.В. Серебряный [14], А.Г. Юрьев [16] и др. Так, в [11] предложено использовать угловую деформацию для описания скачка в угловом перемещении слева и справа от промежуточного шарнира между соседними балками. В [12] использован сложный математический аппарат для получения точного решения контактной задачи об изгибе шарнирно-соединенных балок на упругой полуплоскости. В [15] предложен метод расчета составных балок на упругом основании, однако в нем не учитывается их взаимное влияние. В [14] дано решение пространственной задачи об изгибе шарнирно-соединенных прямоугольных плит на упругом полупространстве и составлены таблицы для облегчения инженерных расчетов. В [16] использован интегральный метод Л.П. Винокурова для решения этой задачи.

Статический расчет шарнирно-соединенных балок на упругом основании Винклера, на упругом полупространстве, а также на

(ㄷ) С. В. Босаков, О. В. Козунова, 2021 
слоистом основании Винклера - Когана был рассмотрен авторами ранее $[1,4,8,9]$. Модель трехслойного упругого основания (модель Винклера - Когана) приводится для расчета изолированной дорожной или аэродромной плиты в [2] и используется для расчета шарнирносоединенных фундаментных и мостовых балок [8, 9]. Отметим, что при исследовании напряженно-деформированного состояния шарнирносоединенных балок на упругом основании Винклера авторами в $[10,17]$ учтена физическая нелинейность материала балок.

Статический расчет шарнирно-соединенных балок на различных моделях упругого основания выполнен при нагрузке от центрально приложенного колеса машины, равномерно распределенной по длине балки. При этом учитывался собственный вес балки.

Из анализа научной литературы по расчету шарнирно-соединенных конструкций на упругом основании можно сделать вывод об отсутствии общего подхода к решению этой проблемы, справедливого для шарнирно-соединенных балок и плит на любой модели упругого основания под действием произвольной внешней нагрузки.

Теория и алгоритм расчета. В данной работе предлагается универсальный подход для расчета шарнирно-соединенных балок на упругом однородном изотропном слое, шарнирно соединенном с недефрормируемым основанием. Он базируется, как и ранее, на смешанном методе строительной механики [13] и способе Жемочкина [7] и позволяет с единых позиций рассчитывать шарнирно-соединенные плиты или балки любой формы и жесткости на различных моделях упругого основания при произвольной вертикальной нагрузке. Численная реализация предлагаемого подхода выполнена для семи шарнирно-соединенных фундаментных балок на упругом слое. Проведено сравнение с аналогичным случаем балок, расположенных на упругом полупространстве.

Этот подход справедлив для балок различной длины и жесткости, на любой модели упругого основания при действии произвольной внешней вертикальной нагрузки. В результате статического расчета шарнирносоединенных балок на упругом основании приводятся: распределение контактных напряжений под конструкцией, поперечные силы в соединительных шарнирах, усилия в связях Жемочкина и перемещения плит или балок.

Постановка задачи. Граничные условия. Рассмотрим систему из семи шарнирно-соединенных балок на упругом основании при действии внешней нагрузки (рис. 1). Требуется определить распределение контактных напряжений под балками, усилия и осадки. 
Вводим следующие условия контактного взаимодействия:

- балки работают совместно с упругим основанием, без отрыва и горизонтального смещения;

- на контакте балки с основанием действуют только нормальные напряжения, силами трения в контактной зоне балки с упругим основанием пренебрегаем (отсутствуют касательные напряжения);

- для балки справедливы гипотезы технической теории изгиба [13];

- распределение контактных напряжений по ширине балки равномерное.

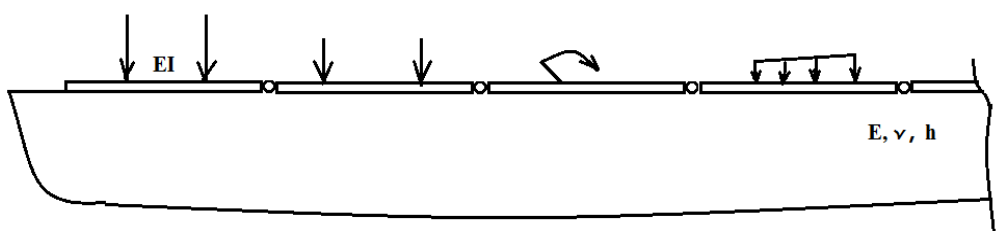

Рис. 1 - Заданная система шарнирно-соединенных балок на упругом однородном изотропном слое

Разобьем каждую балку на участки равной длины и в центре каждого участка поставим вертикальную связь, через которую осуществляется контакт балки с упругим основанием (рис. 2). Полученную многократно статически неопределимую систему решаем смешанным методом строительной механики [13], приняв за неизвестные: усилия $X_{k}$ в связях Б.Н. Жемочкина на контакте балок и основания; линейные и угловые перемещения $u_{k}, \phi_{k}$, введенные на краях балок защемления и поперечные силы $Q_{k}$ в разрезанных промежуточных шарнирах.

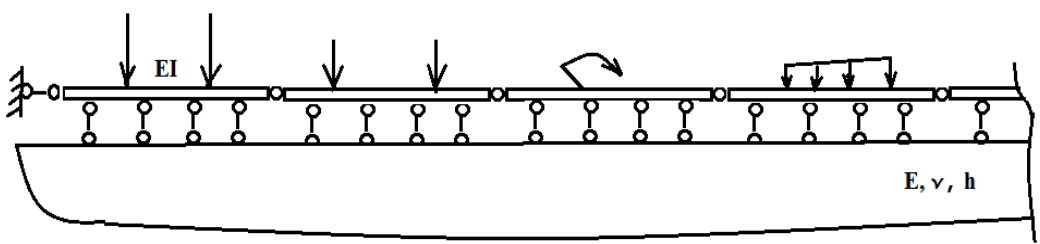

Рис. 2 - Участки и связи Жемочкина

Основная система смешанного метода приведена на рис. 3 .

Система канонических уравнений смешанного метода для расчета одной балки с номером $i$ имеет вид 


$$
\left\{\begin{array}{l}
\delta_{1,1} X_{1}+\ldots+\delta_{1, m} X_{m}+u_{i}-\phi_{i} x_{1}-\delta_{1, Q} Q_{i}+\Delta_{1, p}=0 \\
\ldots \\
\delta_{m, 1} X_{1}+\ldots+\delta_{m, m} X_{m}+u_{i}-\phi_{i} x_{m}-\delta_{m, Q} Q_{i}+\Delta_{m, p}=0 \\
-\sum_{k=1}^{m} X_{k}+Q_{i, 1}+R=0 \\
\sum_{k=1}^{m} X_{k} x_{k}-\ell Q_{i, 1}-M=0 \\
\sum_{k=1}^{m} \delta_{k, Q} X_{k}-\delta_{i, Q} Q_{i}-u_{i}+\ell \phi+\Delta_{Q, p}+u_{i+1}=0,
\end{array}\right.
$$

где $m$-количество участков Б. Н. Жемочкина на балке; $u_{i}, \phi_{i}-$ неизвестные линейное и угловое перемещения введенного защемления на балке с номером $i$ соответственно; $R, M$ - равнодействующая внешних сил и момент равнодействующей относительно введенного защемления на балке с номером $i ; Q_{i}$-поперечная сила в разрезанном шарнире по правую сторону балки с номером $i$; $X_{k}-$ усилие в связи Б. Н. Жемочкина с номером $k$.

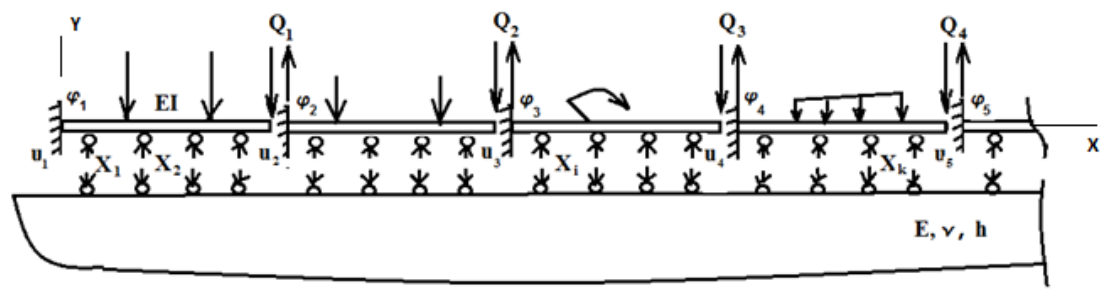

Рис. 3 - Основная система смешанного метода

Для упругого полупространства с модулем упругости $E_{0}$ и коэффрициентом Пуассона $v_{0}$ коэффициенты при неизвестных определяются из соотношения

$$
\delta_{i, k}=\frac{1-v_{0}^{2}}{\pi E_{0} c} F_{i, k}+\frac{\ell^{3}}{3 E I} w_{i, k},
$$

где $E I$ - изгибная жесткость балки; $b$ и $c$ - размеры участка Жемочкина на балке ( $b$ - ширина балки) ( далее они обозначаются через $\Delta y$ и $\Delta x$ соответственно); $F_{i, k}$ - безразмерная фрункция, представляющая 
перемещения середины участка с номером $i$ от единичной силы, распределенной по участку с номером $k$.

Безразмерная функция $F_{i, k}$ в формуле (2) определяется через соотношения из [7]:

$$
\begin{aligned}
& F_{i, i}=2 \frac{c}{b}\left[\ln \frac{b}{c}+\frac{b}{c} \ln \left(\frac{c}{b}+\sqrt{\frac{c^{2}}{b^{2}}+1}\right)+\ln \left(1+\sqrt{\frac{c^{2}}{b^{2}}+1}\right)\right] ; \\
& F_{i, k}=\frac{1}{\left|x_{i}-x_{k}\right|} .
\end{aligned}
$$

Прогибы балки с её защемлением на краю в основной системе смешанного метода определяются по следующим формулам $[7,13]$

$$
\begin{array}{ll}
w_{i, k}=\frac{x_{i}^{2}}{\ell^{2}}\left(3 \frac{x_{k}}{\ell}-\frac{x_{i}}{\ell}\right), & x_{k}>x_{i} ; \\
w_{i, k}=\frac{x_{k}^{2}}{\ell^{2}}\left(3 \frac{x_{i}}{\ell}-\frac{x_{i}}{\ell}\right), & x_{k}<x_{i}
\end{array}
$$

Свободные члены системы (1) зависят от вида внешней нагрузки и определяются с использованием фрормул (4). Отметим, что последнее уравнение в (1) выражает условие отсутствия взаимного вертикального перемещения в промежуточном шарнире между соседними балками.

Для упругого однородного изотропного слоя, шарнирно-соединенного с недеформируемым основанием, вертикальные перемещения поверхности упругого слоя от сосредоточенной силы $P$ определяются через соотношение (2.13) из [3]

$$
W(R)=\frac{P\left(1-v_{0}^{2}\right)}{\pi E_{0}}\left[\frac{1}{R}+\frac{1}{h} \sum_{n=0}^{\infty} a_{n} \frac{\Gamma(n+1)}{\left(4+\frac{R^{2}}{h^{2}}\right)^{\frac{n+1}{2}}} P_{n}\left(\frac{2 h}{\sqrt{R^{2}+4 h^{2}}}\right)\right],
$$

где $R=\sqrt{x^{2}+y^{2}}$ - радиус-вектор перемещающихся точек с координатами $(x, y)$ исследуемой поверхности упругого слоя; $h$ - мощность (толщина) упругого слоя; $a_{n}$ - неопределенные коэффициенты разложения в ряд; $\Gamma(n+1)$ - гамма-фрункция [6]; $P_{n}\left(2 h / \sqrt{R^{2}+4 h^{2}}\right)$ - полином Лежандра [6]. 
В [3] $a_{n}$ были определены: $a_{0}=-1 ; a_{1}=-3 / 2 ; \quad a_{2}=-1$; $a_{3}=-1 / 3 ; a_{4}=1 / 18 ; \ldots$

После интегрирования (5) по площади прямоугольного участка размерами $\Delta x \times \Delta y$ получаем выражения для определения перемещений центра участка Б. Н. Жемочкина с номером $i$ от действия сосредоточенной силы, равной 1 , приложенной к центру участка с номером $k$.

Первое слагаемое в формуле (5) определяет функцию вертикальных перемещений для упругого однородного изотропного полупространства (решение Буссинеска), интегрируется точно (оно сингулярное), остальные - не сингулярные и не интегрируются. Для практических расчетов в формуле (5) можно ограничиться пятью членами ряда [3]. $M\left(x_{i}, y_{i}\right)$

В [3] получено следующее соотношение для перемещения точки

$$
\begin{aligned}
W\left(x_{i}, y_{i}\right) & =\frac{1-v_{0}^{2}}{\pi E_{0} \Delta x}\left[\frac{y_{i}-d}{\Delta y} \ln \frac{x_{i}-b+\sqrt{\left(x_{i}-b\right)^{2}+\left(y_{i}-d\right)^{2}}}{x_{i}-a+\sqrt{\left(x_{i}-a\right)^{2}+\left(y_{i}-d\right)^{2}}}+\right. \\
+ & \frac{y_{i}-c}{\Delta y} \ln \frac{x_{i}-a+\sqrt{\left(x_{i}-a\right)^{2}+\left(y_{i}-c\right)^{2}}}{x_{i}-b+\sqrt{\left(x_{i}-b\right)^{2}+\left(y_{i}-c\right)^{2}}}+ \\
+ & \frac{x_{i}-b}{\Delta y} \ln \frac{y_{i}-d+\sqrt{\left(x_{i}-b\right)^{2}+\left(y_{i}-d\right)^{2}}}{y_{i}-c+\sqrt{\left(x_{i}-b\right)^{2}+\left(y_{i}-c\right)^{2}}}+ \\
+ & \left.\frac{x_{i}-a}{\Delta y} \ln \frac{y_{i}-c+\sqrt{\left(x_{i}-a\right)^{2}+\left(y_{i}-c\right)^{2}}}{y_{i}-d+\sqrt{\left(x_{i}-a\right)^{2}+\left(y_{i}-d\right)^{2}}}\right]+ \\
+ & \left.\frac{1-v_{0}^{2}}{\pi E_{0} h} \sum_{n=0}^{\infty} a_{n} \frac{\Gamma(n+1)}{\left(4+\frac{\left(x_{i}-x_{k}\right)^{2}+\left(y_{i}-y_{k}\right)^{2}}{h^{2}}\right.}\right)^{\times P_{n}\left(\frac{2 h}{\sqrt{\left(x_{i}-x_{k}\right)^{2}+\left(y_{i}-y_{k}\right)^{2}+4 h^{2}}} \times\right.}
\end{aligned}
$$

Запишем соотношение (6) через безразмерную фрунцию $F_{i, k}$, а именно

$$
W\left(x_{i}, y_{i}\right)=\frac{1-v_{0}^{2}}{\pi E_{0} \Delta x} \cdot F_{i k},
$$




$$
\begin{aligned}
& F_{i k}=\frac{y_{i}-d}{\Delta y} \ln \frac{x_{i}-b+\sqrt{\left(x_{i}-b\right)^{2}+\left(y_{i}-d\right)^{2}}}{x_{i}-a+\sqrt{\left(x_{i}-a\right)^{2}+\left(y_{i}-d\right)^{2}}}+ \\
& +\frac{y_{i}-c}{\Delta y} \ln \frac{x_{i}-a+\sqrt{\left(x_{i}-a\right)^{2}+\left(y_{i}-c\right)^{2}}}{x_{i}-b+\sqrt{\left(x_{i}-b\right)^{2}+\left(y_{i}-c\right)^{2}}}+ \\
& +\frac{x_{i}-b}{\Delta y} \ln \frac{y_{i}-d+\sqrt{\left(x_{i}-b\right)^{2}+\left(y_{i}-d\right)^{2}}}{y_{i}-c+\sqrt{\left(x_{i}-b\right)^{2}+\left(y_{i}-c\right)^{2}}}+ \\
& +\frac{x_{i}-a}{\Delta y} \ln \frac{y_{i}-c+\sqrt{\left(x_{i}-a\right)^{2}+\left(y_{i}-c\right)^{2}}}{y_{i}-d+\sqrt{\left(x_{i}-a\right)^{2}+\left(y_{i}-d\right)^{2}}}+ \\
& +\frac{\Delta x}{h} \sum_{n=0}^{\infty} a_{n} \frac{\Gamma(n+1)}{\left(4+\frac{\left(x_{i}-x_{k}\right)^{2}+\left(y_{i}-y_{k}\right)^{2}}{h^{2}}\right)^{\frac{n+1}{2}}} \times \\
& \times P_{n}\left(\frac{2 h}{\sqrt{\left(x_{i}-x_{k}\right)^{2}+\left(y_{i}-y_{k}\right)^{2}+4 h^{2}}}\right) .
\end{aligned}
$$

Если обозначить количество балок через $N$, то общее количество неизвестных усилий в связях Б. Н. Жемочкина, линейных и угловых перемещений, введенных для защемлений на балках и поперечных сил в промежуточных шарнирах для расчета этой системы составных балок выразится формулой

$$
N(m+2)+N-1
$$

Структура системы разрешающих уравнений для системы балок представлена на рис. 4.

Блоки по главной диагонали образованы по системе (1), побочные блоки являются нулевыми в случае основания Винклера. В случае упругого полупространства и упругого изотропного слоя они характеризуют взаимное влияние балок и определяются с использованием формул (3), (4) и (8) соответственно.

После решения системы разрешающих уравнений для балок по усилиям в связях Б. Н. Жемочкина определяются контактные напряжения под балками и их перемещения. 


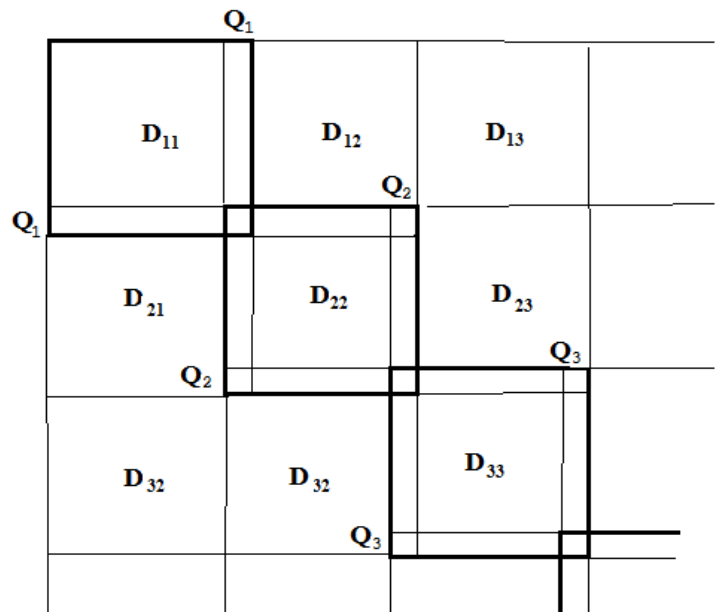

Рис. 4 - Структура системы разрешающих уравнений

Численные результаты. Предлагаемый универсальный подход решения задачи статического расчета шарнирно-соединенных балок при вертикальной нагрузке был численно реализован ранее в $[1,4,8,9]$ на моделях упругого основания Винклера, упругого полупространства и комбинированного основания Винклера - Когана.

В данной работе рассчитается система из семи одинаковых балок длиной 3.0 м и шириной $0.40 \mathrm{M}$, изгибной жесткостью $E I_{\sigma}=2000 \mathrm{kH} \cdot \mathrm{m}^{2}$ на упругом изотропном слое со следующими характеристиками $E_{\text {упр.сл }}=20 M П a ; v=0,3 ; h=3$ м. Балки находятся под действием равномерно распределенной нагрузки. При расчете балка разбивалась на 10 участков Б.Н. Жемочкина.

На рис. 5 приведены графики распределения контактных напряжений под средней балкой для рассматриваемого изотропного слоя. Пунктиром показано аналогичное решение для упругого полупространства.

На рис. 6 и рис. 7 приведены значения поперечных сил в промежуточных шарнирах и вертикальные перемещения для жестких балок на упругом слое от действия симметрично приложенной на среднюю балку сосредоточенной силы $R$. 
Напряжения, $\frac{q L}{b c}$

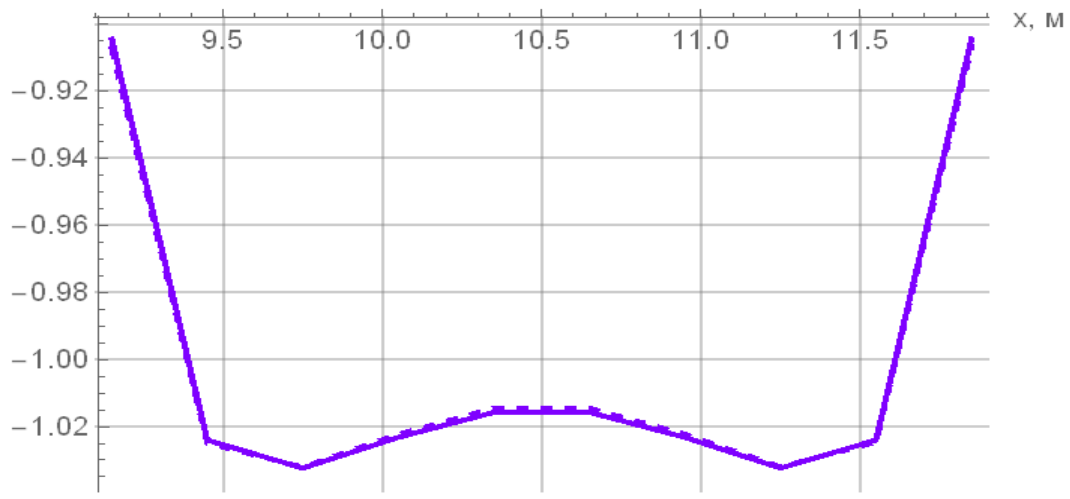

Рис. 5 - Распределение контактных напряжений под средней балкой для упругого изотропного слоя и упругого полупространства

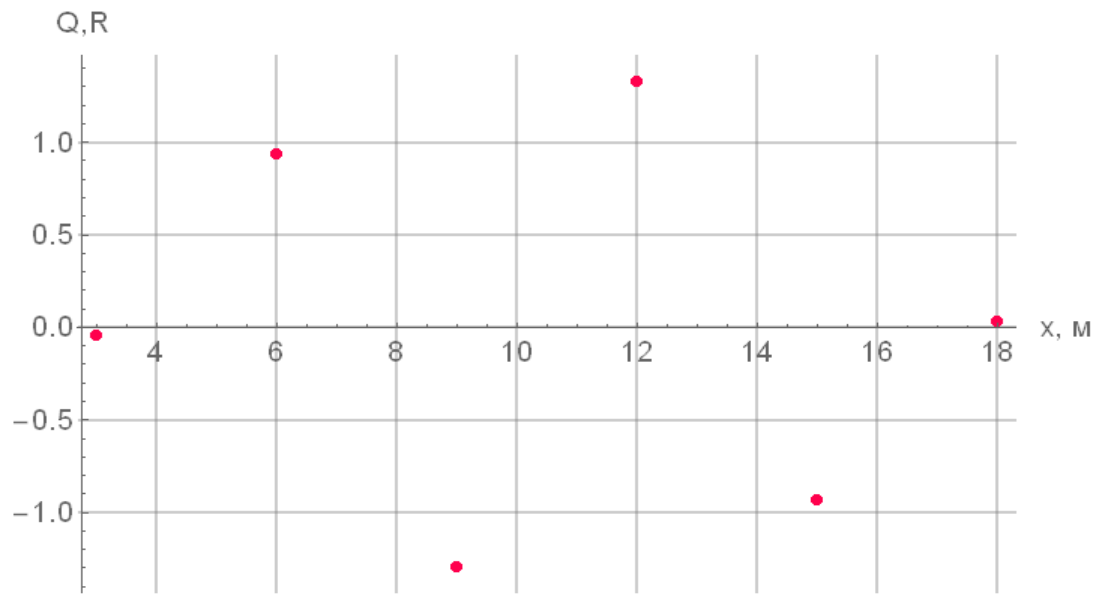

Рис. 6 -Значения поперечных сил в промежуточных шарнирах от сосредоточенной силы $\boldsymbol{R}$ 


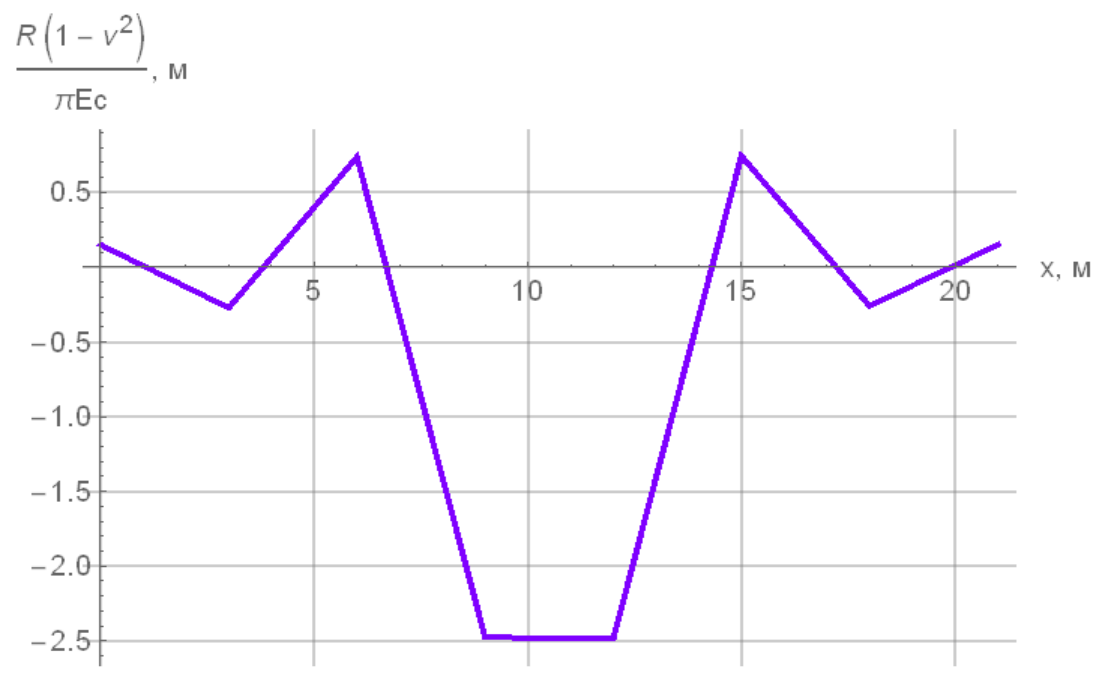

Рис. 7 - График вертикальных перемещений жестких балок от симметрично приложенной на среднюю балку сосредоточенной силы $\boldsymbol{R}$

Выводы. В работе предложена универсальная методика решения контактных задач для системы шарнирно-соединенных балок на упругом основании, моделируемом упругим полупространством и упругим однородным изотропным слоем. Методика основана на смешанном методе строительной механики с использованием способа Жемочкина и может быть обобщена на любое количество балок конечной жесткости и различные модели упругого основания. Отличие от стандартного способа Жемочкина заключается во введении дополнительных неизвестных поперечных сил в соединительных шарнирах и составлении условий равенства вертикальных перемещений в общих шарнирах смежных балок и прямоугольных плит.

Полученные результаты могут быть непосредственно использованы при расчете системы составных фундаментных и мостовых балок, шарнирно-соединенных балочных плит на упругом основании различной инженерно-геологической сложности.

\section{БИБЛИОГРАФИЧЕСКИЕ ССЫЛКИ}

1. Босаков С.В., Зиневич С.И., Козунова О.В. Статический расчет шарнирносоединенных балок на упругом основании // Материалы международной научнотехнической конференции, 23-25 октября 2018. Геотехника Беларуси: наука и практика. Минск: БНТУ, 2018. С. 82-91.

2. Босаков С.В., Зиневич С.И., Козунова О В. Об одной модели упругого основания и ее использовании для расчета прямоугольной плиты на упругом основании // 
НТЖ Строительная механика и расчет сооружений. М., 2018. №4 (279). С. 2-5.

3. Босаков С.В. Статические расчеты плит на упругом основании. Минск: БНТУ, 2002. $128 \mathrm{c}$.

4. Босаков С.В., Козунова О.В. Расчет шарнирно-соединенных балок и плит на упругом основании // Материалы XII Всероссийского съезда по фундаментальным проблемам теоретической и прикладной механике: сборник трудов в 4-х т. Т. 3: Механика деформируемого твердого тела. Уфа: РИЦБашГУ, 2019. С. 873-875.

5. Горбунов-Посадов М.И., Маликова Т.А., Соломин В.И. Расчет конструкций на упругом основании. М.: Стройиздат, 1984. 639 с.

6. Градштейн И.С., Рыжик И.М. Таблицы интегралов, сумм, рядов и произведений. М: Физматлит, 1963. 1098 с.

7. Жемочкин Б. Н., Синицын А. П. Практические методы расчета фундаментных балок и плит на упругом основании. М.: Стройиздат, 1962. 239 с.

8. Козунова О.В. Развитие теории расчета шарнирно-соединенных балок на неоднородном основании // НТЖ: Вестник БрГТУ. 2020. №1. С. 14-21.

9. Козунова О.В. Статический расчет шарнирно-соединенных балок на неоднородном основании // Динамические и технологические проблемы механики конструкций и сплошных сред: материалы XXV Международного симпозиума им. А.Г. Горшкова. Вятичи, 18-22 марта 2019 г. Т.2. Москва: ООО «ТРП». 2019. С. 94-95.

10. Козунова О.В. Учет физической нелинейности шарнирно-соединенных железобетонных балок, расположенных на упругом основании Винклера // Международный сборник научных трудов «Механика. Исследования и инновации». Гомель: БелГУТ, 2019. Вып. 12. С. 121-127.

11. Коренев Б. Г. Вопросы расчета балок и плит на упругом основании. М.: Стройиздат, 1954. 127 с.

12. Попов Г. Я. О расчете неограниченной шарнирно-разрезной балочной плиты, лежащей на упругом полупространстве // Изв. Вузов, Строительство и архитектура. 1959. №3. С. 25-33.

13. Ржаницин А. Р. Строительная механика. М.: Высшая школа, 1991. 439 с.

14. Серебряный $\boldsymbol{P}$. В. Расчет тонких шарнирно-соединенных плит на упругом основании. М.: Стройиздат, 1962. 64c.

15. Симвулиди И.А. Составные балки на упругом основании. М.: Высшая школа, 1961. 204 c.

16. Юрьев А. Г. Балки и плиты с конструктивными и пластическими шарнирами на податливом основании. Харьков: Изв. Вузов. 1964. 46 с.

17. Bosakov S.V., Kozunova O.V. Development of the theory of calculation of hingedconnected beams on an elastic foundation taking into account their physical nonlinearity. Problems of modern concrete and reinforced concrete: collection of articles. scientific. tr. I Institute BeINIIS; editorial board: O.N. Leshkevich [et al.]. Minsk, 2019. Iss. 11. P. 11-24.

УДК 624.13

С. В. Босаков ${ }^{1}$, д-р техн. наук. О. В. Козунова ${ }^{2}$, канд. техн. наук

\section{СТАТИЧНИЙ РОЗРАХУНОК ШАРНІРНО-З'ЄДНАНИХ БАЛОК НА ПРУЖНОМУ ШАРІ}

Статичний розрахунок шарнірно-з'єднаних балок на пружному однорідному ізотропному шарі, як і на інших моделях пружної основи, виконано змішаним методом будівельної механіки і способом Жемочкіна при зосередженому і рівномірно розподіленому навантаженні, що діє на балку 3 урахуванням ії власної ваги. Пропонується універсальний підхід для розрахунку шарнірно- 
з'єднаних балок на пружному шарі, шарнірно з'єднаному з недеформованою основою, що дозволяє 3 єдиних позицій розраховувати шарнірно-з'єднані балки будь-якої жорсткості при довільному вертикальному навантаженні. Числова реалізація пропонованого підходу виконана за допомогою комп'ютерної програми в пакеті Mathematica.

Ключові слова: статичний розрахунок; пружний шар; спосіб Жемочкіна; змішаний метод будівельної механіки.

Теорія розрахунку шарнірних конструкцій на пружній основі на сьогодні ще не повністю розроблена. Дослідження 3 розрахунку шарнірно-з'єднаних балок і плит на пружній основі раніше проводили такі вчені: Б. Г. Коренєв [8], Г. Я. Попов [12], І. А. Сімвуліді [15], Р. В. Серебряний [14], А. Г. Юр'єв [16] та ін. Отже, у [8] запропоновано використовувати кутову деформацію для опису стрибка кутового зміщення вліво і вправо проміжного стику між сусідніми балками. У [12] застосовано складний математичний апарат для отримання точного розв'язку контактної задачі про згинання шарнірно-з'єднаних балок на пружній півплощині. У [15] запропоновано метод розрахунку складених балок на пружній основі, але не враховується взаємовплив балок. У [14] надано розв'язок просторової задачі згинання шарнірних прямокутних пластин на пружному півпросторі та склдено таблиці для полегшення інженерних розрахунків. У [16] використано інтегральний метод Л. П. Вінокурова для вирішення цієї проблеми.

Статичний розрахунок шарнірних балок на пружній основі Вінклера, на пружному напівпросторі та шаруватій основі Вінклера - Когана розглядався авторами раніше $[1,4,9,10]$. Модель тришарової пружної основи (модель Вінклера - Когана) наведена для розрахунку ізольованої дорожньої або повітряно-польової плити у [2] i використовується для розрахунку шарнірно-з'єднаних балок фундаменту та мосту [9, 10]. Слід зазначити, що при дослідженні напруженодеформованого стану шарнірно-з'єднаних балок на пружній основі Вінклера враховано фізичну нелінійність матеріалу балки $[11,17]$.

Статичний розрахунок шарнірно-з'єднаних балок на різних моделях пружної основи виконується при навантаженні від центрально прикладеного колеса машини, рівномірно розподіленому по довжині балки. Враховується власна вага балки.

3 аналізу наукової літератури 3 розрахунку шарнірно-з'єднаних конструкцій на пружній основі можна зробити висновок, що не існує загального підходу до вирішення цієї проблеми, справедливого для шарнірно-з'єднаних балок і плит на будь-якій моделі пружної основи при дії довільного зовнішнього навантаження.

В роботі запропонована універсальна методика розв'язання контактних задач для системи шарнірно-з'єднаних балок на пружній основі, що змодельована пружним півпростором і пружним однорідним ізотропним шаром. Методика заснована на змішаному методі будівельної механіки з використанням способу Жемочкіна і може бути узагальнена на будь-яку кількість балок скінченної жорсткості і різні моделі пружної основи. Відмінність від стандартного способу Жемочкіна 28 
полягає у введенні додаткових невідомих поперечних сил в сполучних шарнірах і складанні умов рівності вертикальних переміщень в загальних шарнірах суміжних балок і прямокутних плит.

Отримані результати можуть бути безпосередньо використані при розрахунку системи складових фрундаментних і мостових балок, шарнірно-з'єднаних балкових плит на пружній основі різної інженерногеологічної складності.

UDC 624.13

\section{S. V. Bosakov', Dr. Sci. (Tech.), O. V. Kozunova², PhD (Tech.) \\ STATIC CALCULATION OF HINGED-CONNECTED BEAMS ON AN ELASTIC LAYER}

The static calculation of hinged-connected beams on an elastic homogeneous isotropic layer, as on other models of an elastic foundation, was carried out in the work by a mixed method of structural mechanics and Zhemochkin's method for a concentrated and uniformly distributed load acting on a beam, taking into account its own weight. Below, a universal approach is proposed for calculating articulated beams on an elastic layer hinged-connected to a non-deformable base. It allows to calculate articulated - connected beams oftheany stiffness for an arbitrary vertical load from a single position. The numerical implementation of the proposed approach was carried out using a computer program in the mathematical package Mathematica.

Keywords: static calculation, elastic layer; Zhemochkin's method; mixed method of structural mechanics.

The theory of calculation of articulated structures on an elastic base has not been fully developed to date. Research on the calculation of articulated beams and plates on an elastic base was previously carried out by the following scientists: B. G. Korenev [8], G. Ya. Popov [12], I. A. Simvulidi [15], R. V. Serebryany [14], A. G. Yuriev [16] and etc.

So, in [8] proposed to use angular deformation to describe the jump in angular displacement to the left and right of the intermediate joint between neighboring beams. In [12] used a complex mathematical apparatus to obtain an exact solution to the contact problem of bending articulated beams on an elastic half-plane. In [15] proposed a method for calculating composite beams on an elastic base, but it does not take into account the mutual influence of beams. In [14] gave a solution to the spatial problem of bending articulated rectangular plates on an elastic half-space and compiled tables to facilitate engineering calculations. In [16] used the integral method of L. P. Vinokurov to solve this problem.

The static calculation of articulated beams on the elastic Winkler base, on an elastic half-space, and on the layered Winkler - Kogan base was considered by the authors earlier [1, 4, 9, 10]. The model of the three-layer elastic base (Winkler - Kogan model) is given for calculating an isolated road or airfield plate in [2], and is used for calculating hinged-connected Foundation and bridge beams [9, 10]. It should be noted that in the study of the stress-strain state of articulated beams on the elastic Winkler base, the 
authors took into account the physical nonlinearity of the beam material in their articles $[11,17]$.

Static calculation of articulated beams on various models of elastic base is performed on the load from the centrally applied machine wheel, evenly distributed along the length of the beam. The beam own weight was taken into account.

From the analysis of scientific literature on the calculation of articulated structures on an elastic base, we can conclude that there is no general approach to solving this problem, which is fair for articulated beams and plates on any model of an elastic base under the action of an arbitrary external load.

The paper proposes a universal technique for solving contact problems for a system of articulated beams on an elastic base, modeled by an elastic half-space and an elastic homogeneous isotropic layer. The technique is based on a mixed method of structural mechanics using the Zhemochkin method and can be generalized to any number of beams of finite stiffness and various models of an elastic foundation. The difference from the standard Zhemochkin method lies in the introduction of additional unknown shear forces in the connecting hinges and in drawing up conditions for the equality of vertical displacements in the common hinges of adjacent beams and rectangular slabs.

The results obtained can be directly used in the calculation of a system of composite foundation and bridge beams, hinged-connected beam slabs on an elastic foundation of various engineering-geological complexity.

\section{REFERENCES}

1. Bosakov S.V., Zinevich S.I., Kozunova O.V. Static calculation of hinged-connected beams on an elastic foundation // Materials of the international scientific and technical conference, October 23-25, 2018. Geotechnics of Belarus: science and practice. Minsk: BNTU, 2018. P. 82-91. (in Russian).

2. Bosakov S.V., Zinevich S.I. Kosunova O.V. A model for the elastic Foundation and its use for the calculation of rectangular plates on elastic foundations / / Scientific-technical journal: Structural mechanics and calculation of structures. Moscow. 2018. No 4 (279). P. 2-5. (in Russian).

3. Bosakov S.V. Static calculations of plates on an elastic base. Minsk: BNTU, 2002. 128 p. (in Russian).

4. Bosakov S.V., Kozunova O.V. Calculation of articulated beams and plates on an elastic foundation // Materials of the XII All-Russian Congress on fundamental problems of theoretical and applied mechanics: a collection of works in 4 volumes. Vol.3: Mechanics of a deformable solid. Ufa: RITSBashGU, 2019. P. 873-875. (in Russian).

5. Gorbunov-Posadov M. I., Malikova T. A., Solomin, V. I. Analysis of structures on elastic Foundation. Moscow: Stroyizdat, 1984. 639 p. (in Russian).

6. Gradshtein I.S., Ryzhik I.M. Tables of integrals, sums, series and products. Moscow: Fizmatlit, 1963. 1098 p. (in Russian).

7. Zhemochkin B. N., Sinitsyn. A. P. Practical methods of calculation of Foundation beams and plates on an elastic base. Moscow: Stroyizdat, 1962. 239 p. (in Russian).

8. Kozunova O.V. Development of the theory of calculation of hinged-connected beams on a non-uniform base // NTZh: Vestnik BrSTU.. 2020. No. 1. P. 14-21. (in Russian). 
9. Kozunova O.V. Static calculation of articulated-connected beams on a nonuniform base // Dynamic and technological problems of structural mechanics and continuous media: materials of the XXV International Symposium named after A.G. Gorshkov. Vyatichi, March 18-22, 2019 .Vol.2. Moscow: LLC TRP. 2019. P. 94-95. (in Russian).

10. Kozunova O.V. Accounting for the physical nonlinearity of articulated reinforced concrete beams located on the elastic foundation of Winkler. // International collection of scientific papers "Mechanics. Research and innovation". Gomel: BeISUT. 2019. Iss.12. P. 121-127. (in Russian).

11. Korenev B. G. Questions of calculation of beams and plates on the elastic basis. Moscow: Stroyizdat, 1954. 127 p. (in Russian).

12. Popov G.Ya. On the calculation of an unbounded hinged-split beam plate lying on an elastic half-space // WPI. Universities, Construction and architecture. 1959. No 3. P. 2533. (in Russian).

13. Rzhanitsin A.R. Structural mechanics. Moscow: High school, 1991. 439 p. (in Russian).

14. Serebryany R.B. Calculation of thin hinged plates on an elastic base. Moscow: Stroyizdat, 1962.64 p. (in Russian).

15. Simvulidi I.A. Composite beams on an elastic foundation. Moscow: Higher school, 1961. 204 p. (in Russian).

16. Yuriev A.G. Beams and plates with constructive and plastic hinges on compliant substrate. Kharkov: Ed. WPI. Higher educational, 1964. 46 p.(in Russian).

17. Bosakov S.V., Kozunova O.V. Development of the theory of calculation of hingedconnected beams on an elastic foundation taking into account their physical nonlinearity. Problems of modern concrete and reinforced concrete: collection of articles. scientific. tr. / Institute BeINIIS; editorial board: O.N. Leshkevich [et al.]. Minsk, 2019. Iss. 11. P. 11-24.

\author{
${ }^{1}$ Белорусский национальный \\ технический университет, \\ Минск, Республика Беларусь \\ ${ }^{2}$ УО «Белорусский государственный \\ университет транспорта», \\ Гомель, Республика Беларусь
}

